\title{
BMJ Open Development of the Brussels Infant and Toddler Stool Scale ('BITSS'): protocol of the study
}

\author{
Yvan Vandenplas, ${ }^{1}$ Hania Szajewska, ${ }^{2}$ Marc Benninga, ${ }^{3}$ Carlo Di Lorenzo, ${ }^{4}$ \\ Christophe Dupont, ${ }^{5}$ Christophe Faure, ${ }^{6}$ Mohamed Miqdadi, ${ }^{7}$ Seksit Osatakul, ${ }^{8}$ \\ Carmen Ribes-Konickx, ${ }^{9}$ Miguel Saps, ${ }^{4}$ Raanan Shamir, ${ }^{10}$ Annamaria Staiano, ${ }^{11}$ \\ on behalf of the BITSS Study Group
}

To cite: Vandenplas $\mathrm{Y}$, Szajewska H, Benninga M, et al. Development of the Brussels Infant and Toddler Stool Scale ('BITSS'): protocol of the study. BMJ Open 2017;7: 014620 . doi:10.1136/bmjopen-2016014620

- Prepublication history for this paper is available online. To view these files please visit the journal online (http://dx.doi.org/10.1136/ bmjopen-2016-014620).

YV and HS are joint lead authors.

Received 11 October 2016 Revised 13 February 2017 Accepted 28 February 2017

\section{CrossMark}

For numbered affiliations see end of article.

\footnotetext{
Correspondence to Dr Yvan Vandenplas: yvan.vandenplas@uzbrussel. be
}

\begin{abstract}
Introduction: The Bristol Stool Form Scale (BSS) which consists of 7 photographs of different stool forms allows assessment of stool consistency (scale 1 for hard lumps to scale 7 for watery stools), in an objective manner in adults. The BSS is also sometimes used to characterise the stools of infants and young children. Despite its use, there is general agreement among paediatric gastroenterologists that the BSS is not adequate to be used in infants and young children who wear diapers; thus, a new scale specifically designed for this population is needed. Our aim is to develop a paediatric stool scale, the Brussels Infant and Toddler Stool Scale ('BITSS'), and to evaluate the interobserver agreement of stool assessment with the BITSS between the patient's parent and healthcare providers (physicians and nurses).
\end{abstract}

Methods and analysis: This study has two phases. In the first phase, 11 key-opinion leaders in the field of paediatric gastroenterology representing different areas of the world selected seven coloured photographs of infants and/or young children wearing diapers to match the original descriptors of the BSS. The selected photographs were used to create a new scale in which the drawings of stools of the BSS were replaced by infant/toddlers stool photographs. In phase II, we aim at demonstrating that parents, nurses and primary healthcare physicians interpret the stool-pictures of the BITSS with a high degree of consensus and that the agreement is independent of whether it is a parent or a healthcare provider. Interobserver variability of stool assessment with the BITSS between the patient's parent and healthcare providers will be assessed.

Ethics and dissemination: The study will be approved by the Ethics Committee of the participating centres. The findings of this study will be submitted to a peer-reviewed journal. Abstracts will be submitted to national and international conferences.

Trial registration number: NCT02913950.

\section{INTRODUCTION}

It has been long thought in the medical community that the patients' or caregivers' description of stool consistency was highly subjective and poorly reliable. Thus, in 1992,

\section{Strengths and limitations of this study}

- Every attempt to classify the form of stools has its limitations. The current lack of a 'gold standard' tool to assess stool consistency makes it prone to subjective interpretation.

- The Brussels Infant and Toddler Stool Scale will be a new tool to describe in an objective way stool consistency in non-toilet-trained infants and young children.

- Worldwide consensus between experts, primary healthcare physicians, nurses and parents will be obtained.

the Bristol Stool Form Scale (hereafter, this scale is referred to as the BSS) was developed to provide an objective way of describing and reporting stool consistency in adults. ${ }^{1}{ }^{2}$ In 1999, the Bristol Stool Chart was added. ${ }^{1}$ The BSS soon became the gold standard to classify stool consistency. In its present form, the 7-point BSS consists of 7 drawings of different stool forms representing a wide range of stool consistencies (scale 1 for hard lumps to scale 7 for watery stools) (see figure 1). The BSS has excellent reliability and agreement when used to rate individual stool type by different individuals. $^{2}$ However, the BSS reliability and agreement decreases when applied to Rome III stool form categories. ${ }^{3-5}$ A modified BSS was shown to be reliable when used also in toilet-trained children 6 years and older. ${ }^{4}$

In 2009, the Amsterdam Stool Scale (hereafter, the Amsterdam Scale) was developed and validated, providing additional information on colour and volume of the defecations of non-toilet-trained children and infants. ${ }^{6}$ In brief, it consists of three visual descriptive scales assessing stool consistency (4-point scale: watery, soft, formed, and hard), amount (4-point scale) and colour (six categories). Compared with the BSS, the Amsterdam Scale was shown to be more suitable for use 


\section{Bristol Stool Chart}

\begin{tabular}{|l|l|}
\hline Type I & $\begin{array}{l}\text { Separate hard lumps, like nuts } \\
\text { (hard to pass) }\end{array}$ \\
\hline Type 2 & Sausage-shaped but lumpy \\
\hline Type 3 & $\begin{array}{l}\text { Like a sausage but with cracks on } \\
\text { its surface }\end{array}$ \\
\hline Type 4 & $\begin{array}{l}\text { Soft blobs with clear-cut edges } \\
\text { (passed easily) }\end{array}$ \\
\hline Type 5 & $\begin{array}{l}\text { Fluffy pieces with ragged edges, a } \\
\text { mushy stool }\end{array}$ \\
\hline Type 6 & $\begin{array}{l}\text { Watery, no solid pieces. } \\
\text { Entirely Liquid }\end{array}$ \\
\hline
\end{tabular}

Figure 1 Illustration of the Modified Bristol Stool Scale. ${ }^{2}$

in infants. Still, the Amsterdam Scale is not universally used; it is too complex and too complicated for routine use by parents, nurses and primary healthcare physicians. $^{\text {p }}$

A recent study showed that among parents of infants and toddlers only fair agreement existed between the BSS and parental report of stool consistency. Moreover, it was documented that different methods of assessment of stool consistency did not result in a difference in the prevalence of functional constipation. ${ }^{8}$ Since there is no better alternative, worldwide, the BSS is used to describe the stools of infants and young children. However, there is consensus among paediatric gastroenterologists that the BSS is not ideal for use in infants and young children who use diapers; thus, a new scale is needed.

\section{OBJECTIVE}

To develop a paediatric stool form scale and to evaluate the interobserver reliability of stool assessment with the paediatric stool form scale, hereafter referred to as the Brussels Infants and Toddlers Stool Scale (BITSS), between the patient's parent and healthcare providers.

\section{METHODS AND ANALYSIS}

This study has two phases. In the first phase, which was completed between February and April 2016, the drawings of adult stools used in the BSS were replaced by colour photographs of the stools of infants and/or young children, not toilet trained, thus wearing diapers.

For this, between February and April 2016, the principal investigator (YV) asked nurses to take photographs of the stools of hospitalised infants and young children. Three cameras were provided to the nurses. After parental consent was obtained, nurses took photographs of diapers containing fresh stools. The type of feeding was not considered as an exclusion or inclusion criterion. Diapers with evidence of melena or fresh blood or mucus were excluded. Then, 28 clear photographs best matching the 7 original descriptions and illustrations used in the original adult BSS were selected during 4 voting sessions with 12 nurses, 2 staff members of the ward and the principal investigator. For each of the 7 original BSS descriptions, the 4 best matching photographs were selected. One hundred per cent agreement was reached in the fourth anonymous voting round. When the photos were grouped, photos 1 and 2 indicated hard stools; photos 3 and 4 indicated normal formed stool; photo 5 indicated loose stools and photos 6 and 7 indicated watery stools.

These 28 pictures were sent out to a first group of investigators (HS, AS, CR-K, CD and RS) who selected the 7 'pictures' best representing the original descriptions of the BSS. After two voting rounds, $100 \%$ full consensus was reached.

The principal investigator then mixed the order of the 7 selected photos and sent them, in combination with the original descriptions and illustrations of the BSS, to $11 \mathrm{key}-$ opinion leaders in the field on paediatric gastroenterology representing different parts of the world. Each colleague was asked to identify the 'best picture' matching each of the BSS descriptions. The first voting round revealed a small interobserver variability (Fleiss $\kappa$ value $=0.86$ ).

In phase II, we plan to demonstrate that parents, nurses and primary healthcare physicians interpret the stool-photographs with a high degree of consensus and that a high degree of consensus can be achieved regardless of whether a parent or a healthcare provider rates the stools. In order to achieve this goal, we plan to evaluate interobserver variability of stool assessment with the BITSS between the patient's parents and healthcare providers. In most countries, the BSS has been translated into local languages. Otherwise, the local investigator will provide an appropriate translation. The protocol for this study is registered at https://register.clinicaltrials.gov.

\section{PARTICIPANTS}

All authors and members of the BITSS study group agreed to enrol a minimum of 50 parents, 25 nurses and 25 primary healthcare physicians, up to a maximum of 100 individuals (participating centres: see list of coauthors and members of BITSS study group). As a result, data will be obtained from at least 500 parents, 250 nurses and 250 physicians, distributed worldwide. The number of parents, nurses and physicians per centre was arbitrarily chosen.

\section{OUTCOME}

The primary outcome of this project is to develop a paediatric version of the BSS, based on a broad 
consensus between healthcare physicians, nurses and parents. Stool composition in non-toilet-trained children is classified according to seven preselected photographs. Stools will be described as follows (figure 1): type 1, 'separate hard lumps'; type 2 'lumpy and sausage like' (types 1 and 2 will be grouped together and considered as 'hard stools'); type 3, 'sausage shaped with cracks on the surface'; type 4, stool in the form of a 'smooth, soft sausage' (types 3 and 4 are grouped together and considered to be 'normal, formed' composition); type 5, 'soft blobs with clear-cut edges', or 'loose' stools, but they are also considered normal, for example in breastfed infants; type 6, 'mushy' consistency, and type 7, entirely liquid consistency with no solid pieces (types 6 and 7 are considered as 'watery stools').

\section{STATISTICAL ANALYSIS}

The interobserver variability of stool assessment between the patient's parents and healthcare providers will be evaluated by calculating the proportion of exact agreement and the Fleiss' $\kappa$ statistics. Correlations, based on the value of $\kappa$, will be categorised as poor $(\kappa \leq 0.2)$, fair $(0.21 \leq \kappa \leq 0.40)$, moderate $(0.41 \leq \kappa \leq 0.60)$, good $(0.61 \leq \kappa \leq 0.80)$ or excellent $(0.81 \leq \kappa \leq 1.00) .{ }^{9}$ Fleiss' $\kappa$ is a statistical measure for assessing the reliability of agreement between a fixed number of raters when assigning categorical ratings to a number of items or classifying items. As the Fleiss' $\kappa$ value decreases with an increasing number of raters, a 'moderate' $\kappa$ value $(>0.41$ to 0.60$)$ is accepted as indicative of a correlation that is high enough to be accepted. The Fleiss' $\kappa$ contrasts with other $\kappa s$ such as Cohen's $\kappa$ that can only be used to assess the agreement between no more than two raters or the intrarater reliability for one appraiser versus himself. The measure calculates the degree of agreement over that would be expected by chance.

The following Fleiss' $\kappa$ values will be calculated: overall, split-up per photograph, split-up per stool group (hard, normal, loose and watery stools), split-up per rater group (physicians, nurses, and parents) and split-up per country. In cases in which the Fleiss' $\kappa$ value shows a poor or fair correlation, the reason for this failure will be evaluated and the photographs and/or descriptions will be adapted.

\section{DISSEMINATION}

The findings of this study will be submitted to a peerreviewed journal. Abstracts will be submitted to relevant national and international meetings.

\section{CONCLUSIONS}

The optimal way of classifying stool consistency in infants and young children not yet toilet trained is still debated. Our multicentre study, carried out at research centres with experience in conducting clinical studies in children with gastrointestinal disorders, intends to address a gap in describing stool consistency, providing a much needed tool for the objective assessment of stool consistency for infants and toddlers wearing diapers.
Author affiliations

${ }^{1}$ Department of Pediatrics, UZ Brussel, Vrije Universiteit Brussel, Brussels, Belgium

${ }^{2}$ Department of Pediatrics, The Medical University of Warsaw, Warsaw, Poland ${ }^{3}$ Department of Pediatrics, Emma Children's Hospital/AMC, Amsterdam, The Netherlands

${ }^{4}$ Division of Pediatric Gastroenterology, Hepatology, and Nutrition, Nationwide Children's Hospital, Columbus, Ohio, USA

${ }^{5}$ Department of Pediatric Gastroenterology Hepatology and Nutrition, Hôpital Necker Enfants Malades, Paris, France

${ }^{6}$ Pediatric Gastroenterology, Sainte-Justine Hospital, Montreal, Quebec, Canada

${ }^{7}$ Sheikh Khalifa Medical City, Abu Dhabi, UAE

${ }^{8}$ Department of Pediatrics, Faculty of Medicine, Prince of Songkla University, Hat Yai, Songkhla, Thailand

${ }^{9}$ Pediatric Gastroenterology and Hepatology Unit, La Fe University Hospital, Valencia, Spain

${ }^{10}$ Schneider Children's Medical Centre of Israel, Sackler Faculty of Medicine, Tel-Aviv University, Tel-Aviv, Israel

${ }^{11}$ Department of Translational Medical Science, Section of Pediatrics, University of Naples Federico II, Naples, Italy

Collaborators The Paediatric Bristol Stool Scale Study Group: Johan Franckx (Belgium), Robin Green (South Africa), Badriul Hegar (Indonesia), Roel Lemmens (Belgium), Silvia Salvatore (Italy), Mario Vieira (Brazil), Marc Verghote (Belgium) and Ioannis Xinias (Greece).

Contributors YV conceptualised the study. YV and HS developed the first draft of the manuscript and contributed equally. All authors contributed to the development of the study protocol and approved the final draft of the manuscript.

Funding The project runs independently from industry, without any funding or financial compensation. All work was and will be carried out 'freelance'.

Competing interests None declared.

Provenance and peer review Not commissioned; externally peer reviewed.

Open Access This is an Open Access article distributed in accordance with the Creative Commons Attribution Non Commercial (CC BY-NC 4.0) license, which permits others to distribute, remix, adapt, build upon this work noncommercially, and license their derivative works on different terms, provided the original work is properly cited and the use is non-commercial. See: http:// creativecommons.org/licenses/by-nc/4.0/

\section{REFERENCES}

1. Heaton KW, Radvan J, Cripps $\mathrm{H}$, et al. Defecation frequency and timing, and stool form in the general population: a prospective study. Gut 1992;33:818-24.

2. Lewis SJ, Heaton KW. Stool form scale as a useful guide to intestinal transit time. Scand J Gastroenterol 1997;32:920-4.

3. Chumpitazi BP, Lane MM, Czyzewski DI, et al. Creation and initial evaluation of a Stool Form Scale for children. J Pediatr 2010;157:594-7.

4. Lane MM, Czyzewski DI, Chumpitazi BP, et al. Reliability and validity of a modified Bristol Stool Form Scale for children. J Pediatr 2011;159:437-41.e1.

5. Chumpitazi BP, Self MM, Czyzewski DI, et al. Bristol Stool Form Scale reliability and agreement decreases when determining Rome III stool form designations. Neurogastroenterol Motil. 2016;28:443-8.

6. Bekkali N, Hamers SL, Reitsma JB, et al. Infant stool form scale: development and results. J Pediatr. 2009;154:521-26.e1.

7. Ghanma A, Puttemans K, Deneyer M, et al. Amsterdam infant stool scale is more useful for assessing children who have not been toilet trained than Bristol stool scale. Acta Paediatr 2014;103: e91-2.

8. Koppen IJ, Velasco-Benitez CA, Benninga MA, et al. Using the Bristol Stool Scale and parental report of stool consistency as part of the Rome III Criteria for functional constipation in infants and toddlers. J Pediatr 2016;177:44-8.e1.

9. Altman DG. Practical statistics for medical research. London: Chapman \& Hall, 1991. 